\title{
The effectiveness of mindfulness-based cognitive therapy for major depressive disorder: evidence from routine outcome monitoring data
}

\author{
Dirk E.M. Geurts*, Felix R. Compen*, Marleen H.C.T. Van Beek and Anne E.M. Speckens
}

\section{Background}

Meta-analyses show efficacy of mindfulness-based cognitive therapy (MBCT) in terms of relapse prevention and depressive symptom reduction in patients with major depressive disorder (MDD). However, most studies have been conducted in controlled research settings.

\section{Aims}

We aimed to investigate the effectiveness of MBCT in patients with MDD presenting in real-world clinical practice. Moreover, we assessed whether guideline recommendations for MBCT allocation in regard to recurrence and remission status of MDD hold in clinical practice.

\section{Method}

This study assessed a naturalistic cohort of patients with (recurrent) MDD, either current or in remission $(n=765)$, who received $\mathrm{MBCT}$ in a university hospital out-patient clinic in The Netherlands. Outcome measures were self-reported depressive symptoms, worry, mindfulness skills and self-compassion. Predictors were MDD recurrence and remission status, and clinical and sociodemographic variables. Outcome and predictor analyses were conducted with linear regression.

\section{Results}

MBCT adherence was high (94\%). Patients with a lower level of education had a higher chance of non-adherence. Attending more sessions positively influenced improvement in depressive symptoms. Depressive symptoms significantly reduced from pre- to post-MBCT ( $\Delta$ mean $=7.7,95 \% \mathrm{Cl}=7.0-8.5$, cohen's $d=0.75$ ). Improvement of depressive symptoms was independent from MDD recurrence and remission status. Unemployed patients showed less favourable outcomes. Worry, mindfulness skills and self-compassion all significantly improved. These improvements were related to changes in depressive symptoms.

\section{Conclusions}

Previous efficacy results in controlled research settings are maintained in clinical practice. Results illustrate that MBCT is effective in routine clinical practice for patients suffering from MDD, irrespective of MDD recurrence and remission status.

\section{Keywords}

Depressive disorders; group psychotherapy; outcome studies; out-patient treatment; comorbidity.

\section{Copyright and usage}

(C) The Author(s), 2020. Published by Cambridge University Press on behalf of The Royal College of Psychiatrists. This is an Open Access article, distributed under the terms of the Creative Commons Attribution-NonCommercial-NoDerivatives licence (http://creativecommons.org/licenses/by-nc-nd/4.0/), which permits non-commercial re-use, distribution, and reproduction in any medium, provided the original work is unaltered and is properly cited. The written permission of Cambridge University Press must be obtained for commercial re-use or in order to create a derivative work.
Major depressive disorder (MDD) is the leading cause of ill health and disability worldwide, and a major contributor to the overall global burden of disease. ${ }^{1,2}$ The frequently recurrent course of MDD is a great contributor to these figures. Mindfulness-based cognitive therapy (MBCT) was specifically developed as a psychological treatment for the prevention of relapse and recurrence of MDD. ${ }^{3}$ Over the years, numerous randomised controlled trials (RCTs) contributed to a strong evidence base demonstrating the efficacy of MBCT in this prevention ${ }^{4}$ and, more recently, in reducing symptoms in those suffering from current MDD. ${ }^{5}$ Notwithstanding this established efficacy of MBCT, there is a large 'implementation gap' in research on MBCT. ${ }^{6}$ Most of the evidence for the efficacy of MBCT comes from RCTs, which are typically conducted in 'ideal' settings (often by the developers of the treatment, with highly qualified teachers) with strict inclusion and exclusion criteria for participant selection, and homogeneous samples as a result. ${ }^{7}$ Psychological treatment effect sizes might decrease substantially when translated from research settings into clinical practice. For example, individual psychological treatments for MDD were estimated to sort only half the effect size in clinical practice compared with the effect size reported in RCTs $\left(0.8 v .1 .71\right.$, respectively) ${ }^{8}$ As

* Joint first authors.
MBCT holds promise as a safe, efficacious ${ }^{4}$ and cost-effective ${ }^{9}$ treatment for MDD and is increasingly implemented in the UK, ${ }^{10}$ there is a need to know what effects of MBCT can be expected in patients with established MDD in clinical practice.

Based on early findings, ${ }^{3,11}$ national clinical guidelines only advise MBCT for those with three or more previous depressive episodes, assuming that those with less prior episodes would not benefit from MBCT. ${ }^{12}$ However, reduction of residual symptoms by MBCT was recently shown to be independent of the number of previous episodes of MDD. ${ }^{13}$ In addition, two meta-analyses including 500 and 1000 patients, demonstrated efficacy of MBCT also in patients with current depressive symptoms independent of recurrence status. ${ }^{5,14}$ For efficient therapy allocation it is necessary to find out whether MBCT is effective for patients with MDD with different recurrence (recurrent versus single) and remission status (current versus remitted) in clinical practice. Thus, we assessed the effectiveness of MBCT in terms of depressive symptom reduction in a large, naturalistic uncontrolled sample of patients diagnosed with MDD with different recurrence and remission status of MDD. The main outcome of interest was the amount of post-MBCT depressive symptoms as a function of recurrence and remission status of MDD. A secondary aim was to inform clinical practice by evaluating possible demographic and clinical predictors of MBCT adherence and outcome. In addition, we assessed whether changes in depressive 
symptoms were associated with changes in potential mediators such as worry, mindfulness skills and self-compassion.

\section{Method}

\section{Design}

The study sample consisted of a naturalistic uncontrolled cohort of patients who received MBCT at the Radboud University Medical Centre for Mindfulness in Nijmegen, The Netherlands, between July 2012 and April 2018. Patients were referred to this setting by either general practitioners or their attending psychologists or psychiatrists. As part of the routine outcome monitoring, patients were asked to complete a set of self-reported questionnaires (see below) before the start of MBCT. After the final MBCT session, patients were asked to complete the questionnaires for a second time. Only those patients who filled in the questionnaires at least at one of the two time points were included in the current study.

\section{Procedure}

All referred patients received a clinical assessment before taking part in the MBCT, which included a semi-structured psychiatric interview (see below). The attending psychiatrist evaluated whether MBCT was suitable for the patient or whether other evidencebased treatments were preferable. Current substance dependency, acute suicidality and acute psychotic symptoms were grounds for excluding patients from MBCT and referring them to other treatments. Moreover, motivation and practical barriers for attending MBCT were discussed. Patients were invited to participate in MBCT when they were willing to participate in a group setting, adhere to homework assignments and able to attend at least six out of eight sessions and the silent day. The psychiatric history, assessment and treatment plan were summarised in a written report, which was subsequently sent to the referrer and the patient. Informed consent for the routine outcome monitoring was obtained via an opt-out system, meaning that patients were informed about the possibility that their anonymised outcome data could be used in scientific research. We received approval from the ethical committee of Radboud University Medical Center to use these anonymised data for research purposes (approval number CMO 2015 1972).

\section{Intervention}

In accordance with the MBCT protocol originally developed by Segal, Williams and Teasdale, ${ }^{15} \mathrm{MBCT}$ consisted of eight weekly 2.5 -h sessions, a silent day between session six and seven, and home assignments for 30-45 min per day. Each MBCT group consisted of 8-12 participants. MBCT was taught to heterogeneous patient groups, mostly consisting of patients with unipolar (recurrent) MDD, either currently depressed or in (partial) remission, but also including patients with comorbid anxiety disorder, attention-deficit hyperactivity disorder, autism and/or personality disorder. The MBCT sessions consisted of guided meditation exercises, psychoeducation, and dialogue and inquiry about the exercises. The courses were taught by qualified teachers meeting the advanced criteria of the Association of Mindfulness-Based Teachers in The Netherlands and Flanders, which are in concordance with the good practice guidelines of the UK Network for Mindfulness-Based Teachers. ${ }^{16}$

\section{Measures}

Demographic and clinical variables

Demographic (gender, age, work status, education level) and clinical variables (psychiatric and somatic comorbidity) were extracted from the electronic patient health record. Work status was recoded into 'employed' (classification: currently paid work, student, homemaker, retired), 'sick leave' and 'unemployed' (job-seeking, long-term disability, social services). Education level was recoded into 'lower' (no education, primary education, prevocational secondary education), 'intermediate' (lower general secondary education, secondary vocational education) and 'higher' (higher secondary general/pre-university education, higher professional education and university education). Chronic somatic diseases were classified in accordance with The Netherlands Study of Depression in Older Persons (NESDO): ${ }^{17}$ lung, cardiovascular, stroke, diabetes, arthritis/rheumatism, gastrointestinal, cancer, epilepsy, thyroid dysfunction, hypertension and hypercholesterolemia. Somatic comorbidity was recoded into no, one and more than one comorbid disorder.

\section{Psychiatric diagnosis}

The semi-structured psychiatric diagnostic interview used to determine psychiatric disorder was the Mini International Neuropsychiatric Interview Plus (MINI-Plus). ${ }^{18}$ This was developed to assess patients for psychiatric diagnosis according to DSM-IV-TR criteria. The instrument has good psychometric properties and is widely used to make psychiatric diagnoses in clinical practice. The MINI-Plus was conducted by trained psychiatrists and psychologists. In addition to MDD, anxiety, somatisation, dysthymia, developmental, addiction and eating and personality disorders were categorised. Psychiatric comorbidity was recoded into no, one and more than one comorbid disorder.

\section{Self-reported outcome measures}

Depressive symptoms were measured with the 21-item Beck Depression Inventory (BDI-II). ${ }^{19}$ Worry was measured with the 16-item Penn State Worry Questionnaire. ${ }^{20,21}$ Mindfulness skills were measured with the 24-item Five Facet Mindfulness Questionnaire Short Form. ${ }^{22}$ Self-compassion was measured with the 12-item Self-Compassion Scale-Short Form. ${ }^{23}$ The psychometric properties of all instruments are considered to be adequate to good.

\section{Adherence}

Information on the total number of MBCT sessions attended was extracted from the electronic patient health record. The maximum number of sessions was nine (eight sessions and one silent day). In accordance with the convention in studies on $\mathrm{MBCT}^{9}$ (e.g. we categorised patients as non-completers when they missed more than five out of nine sessions).

\section{Data preparation}

Demographic and clinical variables, psychiatric diagnosis and MBCT adherence were manually extracted from the electronic patient records by the authors and four research assistants. Around every 200 entries, coding inconsistencies were resolved by discussion between the authors. Self-report measures were scored using data capture software (TeleForm Elite, Cardiff Software, Version 8.2; see https://www.opentext.nl/). Self-report measures were merged with the demographic and clinical data-set. Data were checked for outliers by visual inspection.

\section{Data analysis}

Data analyses were conducted in open-source statistical software program R (RStudio: Integrated Development for R. R Studio, Inc., Boston, MA (2019); see http://www.rstudio.com/). ${ }^{24}$ Descriptive statistics of demographic and clinical variables of patients were 
calculated and compared with $\chi^{2}$ or ANOVA statistics. Analyses of the primary and secondary research aims were analysed with linear regression and generalised linear model analyses. To assess predictors independent of baseline depressive symptom severity, we used residualised change scores of the BDI-II as the dependent variable. Tested predictors were all the demographic and clinical variables as presented in Table 1. Each predictor was tested in a separate linear model. Within-group effect sizes (Cohen's $d$ ) were calculated by

Table 1 Demographic and baseline clinical data compared between those with a complete pre-to-post data-set and those with either missing pre or missing post data

\begin{tabular}{|c|c|c|c|c|}
\hline & Full data-set $(n=504)$ & Missing $(n=261)$ & Total $(N=765)$ & $P$ value \\
\hline Female & $324(64.3 \%)$ & $161(61.7 \%)$ & 485 (63.4\%) & 0.479 \\
\hline Age, years & & & & 0.002 \\
\hline Mean (s.d.) & $47.7(12.7)$ & 44.7 (13.7) & 46.7 (13.2) & \\
\hline Range & $19.0-78.0$ & $19.0-82.0$ & $19.0-82.0$ & \\
\hline Major depressive disorder & & & & 0.182 \\
\hline Single current & $48(9.5 \%)$ & $22(8.4 \%)$ & $70(9.2 \%)$ & \\
\hline Recurrent current & $180(35.7 \%)$ & 75 (28.7\%) & 255 (33.3\%) & \\
\hline Single remitted & $48(9.5 \%)$ & $26(10.0 \%)$ & 74 (9.7\%) & \\
\hline Recurrent remitted & $228(45.2 \%)$ & $138(52.9 \%)$ & $366(47.8 \%)$ & \\
\hline Psychiatric comorbidity & & & & 0.143 \\
\hline No comorbidity & $256(50.8 \%)$ & 119 (45.6\%) & 375 (49.0\%) & \\
\hline One comorbid disorder & 197 (39.1\%) & $104(39.8 \%)$ & 301 (39.3\%) & \\
\hline Multiple comorbidities & $51(10.1 \%)$ & $38(14.6 \%)$ & 89 (11.6\%) & \\
\hline Anxiety disorder & $120(23.8 \%)$ & $58(22.2 \%)$ & 178 (23.3\%) & 0.622 \\
\hline Somatisation disorder & $36(7.1 \%)$ & $10(3.8 \%)$ & $46(6.0 \%)$ & 0.068 \\
\hline Dysthymia & $31(6.2 \%)$ & $10(3.8 \%)$ & $41(5.4 \%)$ & 0.177 \\
\hline Developmental disorder & 38 (7.5\%) & $50(19.2 \%)$ & $88(11.5 \%)$ & $<0.001$ \\
\hline Addiction & $11(2.2 \%)$ & 9 (3.4\%) & $20(2.6 \%)$ & 0.298 \\
\hline Eating disorder & $10(2.0 \%)$ & $1(0.4 \%)$ & $11(1.4 \%)$ & 0.078 \\
\hline Personality disorder & $54(10.7 \%)$ & $43(16.5 \%)$ & 97 (12.7\%) & 0.023 \\
\hline Somatic comorbidity & & & & 0.482 \\
\hline Missing & 2 & 1 & 3 & \\
\hline No somatic comorbidity & $284(56.6 \%)$ & $154(59.2 \%)$ & $438(57.5 \%)$ & \\
\hline Somatic comorbidity & $218(43.4 \%)$ & $106(40.8 \%)$ & $324(42.5 \%)$ & \\
\hline Education level & & & & 0.193 \\
\hline Missing & 83 & 34 & 117 & \\
\hline Lower & 65 (15.4\%) & $41(18.1 \%)$ & $106(16.4 \%)$ & \\
\hline Intermediate & $74(17.6 \%)$ & 50 (22.0\%) & $124(19.1 \%)$ & \\
\hline Higher & $282(67.0 \%)$ & $136(59.9 \%)$ & 418 (64.5\%) & \\
\hline Work & & & & 0.640 \\
\hline Missing & 39 & 24 & 63 & \\
\hline Employed/student/homemaker & 309 (66.5\%) & 149 (62.9\%) & 458 (65.2\%) & \\
\hline Sick leave & $49(10.5 \%)$ & $28(11.8 \%)$ & 77 (11.0\%) & \\
\hline Unemployed & 107 (23.0\%) & $60(25.3 \%)$ & 167 (23.8\%) & \\
\hline $\mathrm{BDI}-\mathrm{II}$ & & & & 0.097 \\
\hline Missing & 1 & 7 & 8 & \\
\hline Mean (s.d.) & $21.7(10.2)$ & $20.3(11.7)$ & $21.2(10.7)$ & \\
\hline Range & $0.0-54.0$ & $0.0-55.0$ & $0.0-55.0$ & \\
\hline PSWQ & & & & 0.020 \\
\hline Missing & 4 & 10 & 14 & \\
\hline Mean (s.d.) & $59.9(12.0)$ & $57.7(12.9)$ & $59.1(12.4)$ & \\
\hline Range & $22.0-80.0$ & $18.0-80.0$ & $18.0-80.0$ & \\
\hline FFMQ & & & & 0.009 \\
\hline Missing & 6 & 5 & 11 & \\
\hline Mean (s.d.) & $70.2(11.2)$ & 72.5 (11.5) & 71.0 (11.3) & \\
\hline Range & $42.0-107.0$ & $35.0-104.0$ & $35.0-107.0$ & \\
\hline Self-compassion & & & & 0.004 \\
\hline Missing & 10 & 9 & 19 & \\
\hline Mean (s.d.) & $21.1(3.5)$ & $21.9(4.0)$ & $21.4(3.7)$ & \\
\hline Range & $12.0-32.0$ & $11.0-32.0$ & $11.0-32.0$ & \\
\hline Antidepressant medication use & & & & 0.478 \\
\hline Missing & 8 & 3 & 11 & \\
\hline None & $271(54.6 \%)$ & $157(60.9 \%)$ & $428(56.8 \%)$ & \\
\hline MAO-I & $2(0.4 \%)$ & $2(0.8 \%)$ & $4(0.5 \%)$ & \\
\hline TCA & $48(9.7 \%)$ & $16(6.2 \%)$ & $64(8.5 \%)$ & \\
\hline SSRI & $157(31.7 \%)$ & 74 (28.7\%) & 231 (30.6\%) & \\
\hline SNRI & $10(2.0 \%)$ & $5(1.9 \%)$ & $15(2.0 \%)$ & \\
\hline Other & $8(1.6 \%)$ & $4(1.6 \%)$ & $12(1.6 \%)$ & \\
\hline Number of sessions attended & & & & $<0.001$ \\
\hline Mean (s.d.) & $8.4(0.9)$ & $6.7(2.6)$ & $7.8(1.9)$ & \\
\hline Range & $2.0-9.0$ & $1.0-9.0$ & $1.0-9.0$ & \\
\hline More than four sessions attended & $502(99.6 \%)$ & $216(82.8 \%)$ & 718 (93.9\%) & $<0.001$ \\
\hline
\end{tabular}


dividing pre-post MBCT differences in outcomes by the pooled pre-post MBCT s.d. of the respective outcome. In case of significant differences in outcomes between patients with different recurrence (recurrent versus single) and remission (current versus remitted) status of MDD, between-group effect sizes (Cohen's $d$ ) were calculated by dividing the differences in outcomes between groups at post-MBCT by the pooled s.d. at baseline. Cohen's $d$ effect sizes of $0.20,0.50$ and 0.80 were considered small, medium and large, respectively. ${ }^{25}$ In addition, reliable change indices ${ }^{26}$ obtained with Cronbach's alpha ${ }^{27}$ were calculated to report percentages of reliably improved and deteriorated patients.

\section{Results}

\section{Study population}

The study sample included 765 patients with MDD (see Table 1). Of this sample, $81 \%$ had experienced two or more previous episodes of MDD and $42 \%$ were diagnosed with current MDD. Moreover, half of the patients was classified as having one or more comorbid psychiatric disorders. About $25 \%$ of patients suffered from a comorbid anxiety disorder and $13 \%$ were classified as having a personality disorder. Somatic comorbidities were also highly prevalent (43\%) in our population (see Table 1).

Pre- and post-MBCT measurements were available for $64 \%$ $(n=504)$ of the patients. Patients with missing data $(n=262$; missing pre-MBCT, $n=6$; missing post-MBCT, $n=257$ ) were, on average, $3.0(95 \% \mathrm{CI}=1.1-5.1)$ years younger, were more often diagnosed with comorbid developmental or personality disorders and attended, on average, $1.7(95 \% \mathrm{CI}=1.4-2.0)$ fewer sessions than completers (see Table 1). Patients with missing data had slightly lower levels of depressive symptoms and worry, and higher levels of mindfulness skills and self-compassion pre-MBCT.

\section{Adherence}

The average number of attended sessions was 7.8 (range 1-9, s.d. = 1.9; see Table 1). Of the patients, $94 \%$ attended four or more sessions (see Supplementary Table 1 available at https://doi.org/10.1192/bjo. 2020.118). Patients with a lower level of education had $12 \%$ chance to attend less than four sessions, whereas for those with intermediate and higher levels of education, this chance was $5 \%\left(\chi^{2}=7.7\right.$, $P=0.02$ ). None of the other included demographic and clinical measures predicted adherence.

\section{Effectiveness of MBCT}

Patients reported significantly less depressive symptoms post-MBCT compared with before MBCT $(\Delta$ mean $=7.8,95 \% \mathrm{CI}=7.0-8.6$, Cohen's $d=0.75$; see Table 2 and upper panel of Fig. 1). Reliable change analyses ${ }^{26}$ revealed that a total of $40 \%$ of all patients improved and $3 \%$ deteriorated in terms of depressive symptoms (see lower panel of Fig. 1). Worry (Cohen's $d=0.52$ ), mindfulness skills (Cohen's $d=0.63$ ) and self-compassion (Cohen's $d=0.75$ ) also improved significantly.

\section{Effects of MDD recurrence and remission status on depressive symptom reduction}

Linear regression including MDD recurrence (recurrent versus single) and remission status (current versus remitted) as factors showed that the reduction of depressive symptoms did not significantly depend on either MDD recurrence or remission status $\left(F_{(1,498)}=0.9, \quad P=0.35 ; \quad F_{(1,498)}=1.9, \quad P=0.17\right.$, respectively), or their interaction $\left(F_{(1,498)}=0.8, \quad P=0.38\right.$; see Table 3 and Supplementary Fig. 1).

\section{Predictors of MBCT effectiveness}

As expected, baseline severity strongly predicted change in depressive symptoms: about $20 \%$ of variance in change was captured by baseline depressive symptoms $\left(R^{2}=0.2, F_{(1,500)}=123, P=9 \times 10^{-26}\right.$; see Supplementary Fig. 2). Employment status significantly affected treatment success of MBCT over and above baseline depressive symptoms $\quad\left(R^{2}=0.03, \quad F_{(1,460)}=6.8, \quad P=0.001 ; \quad\right.$ Supplementary Fig. 2). Simple contrasts showed that patients who had a daytime job or were on sick leave did not significantly differ from each other in terms of symptom reduction, but both groups improved more than those who were unemployed. In addition, we found small explanatory effects of comorbidity: existence of multiple psychiatric comorbidity $\left(R^{2}=0.011, F_{(1,499)}=3.0, P=0.048\right)$ and somatic problems $\left(R^{2}=0.007, F_{(1,499)}=3.7, P=0.056\right)$ tended to predict less effect of MBCT.

Moreover, attending more sessions positively influenced improvement in depressive symptoms $\left(R^{2}=0.01, F_{(1,500)}=6.2\right.$, $P=0.01)$.

\section{Changes of potential mediators related to depressive symptom reduction}

Decreases in depressive symptoms (residualised change score) were associated with decreases in worry and increases in

\begin{tabular}{|c|c|c|c|c|c|c|c|}
\hline & Pre $(n=504)$ & Post $(n=503)$ & $P$ value & Mean difference & $95 \% \mathrm{Cl}$ low & $95 \% \mathrm{Cl}$ high & Cohen's d \\
\hline BDI-II & & & $<0.001$ & -7.81 & --8.60 & -7.02 & -0.75 \\
\hline Missing & 1 & 1 & & & & & \\
\hline Mean (s.d.) & $21.7(10.2)$ & $13.9(10.2)$ & & & & & \\
\hline Range & $0.0-54.0$ & $0.0-56.0$ & & & & & \\
\hline PSWQ & & & $<0.001$ & -6.37 & -7.21 & -5.53 & -0.52 \\
\hline Missing & 4 & 2 & & & & & \\
\hline Mean (s.d.) & $59.9(12.0)$ & $53.4(12.2)$ & & & & & \\
\hline Range & $22.0-80.0$ & $18.0-80.0$ & & & & & \\
\hline FFMQ & & & $<0.001$ & 7.00 & 6.08 & 7.92 & 0.63 \\
\hline Missing & 6 & 4 & & & & & \\
\hline Mean (s.d.) & $70.2(11.2)$ & 77.4 (11.0) & & & & & \\
\hline Range & 42.0-107.0 & $41.0-112.0$ & & & & & \\
\hline Self-compassion & & & $<0.001$ & 2.72 & 2.42 & 3.01 & 0.75 \\
\hline Missing & 10 & 7 & & & & & \\
\hline Mean (s.d.) & $21.1(3.5)$ & $23.9(3.5)$ & & & & & \\
\hline Range & $12.0-32.0$ & $12.0-35.0$ & & & & & \\
\hline
\end{tabular}



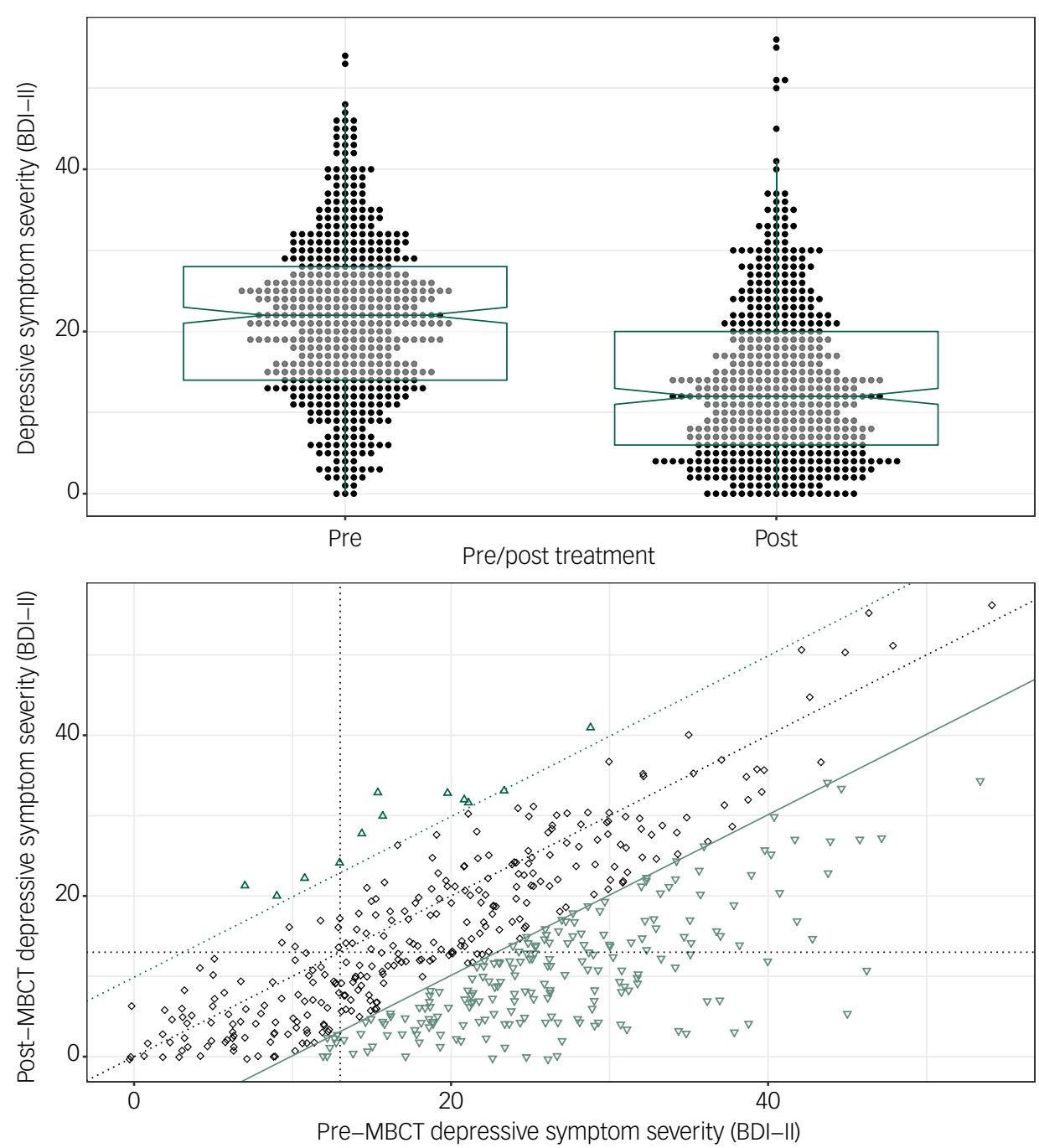

Fig. 1 Change in depressive symptoms based on Beck depression inventory II (BDI-II) between pre- and post-mindfulness-based-cognitivetherapy (MBCT). In the upper panel changes across the whole group $(n=504)$ are depicted. The diagonal line in the lower panel represents 'no pre-post measurement BDI-II change' and the dashed upper and lower lines represent the bounds of the $95 \% \mathrm{Cl}$ of the Jacobson-Truax Reliable Change Index. See text for accompanying numbers and percentages.

Down-pointing triangle, patients who reliably improved; up-pointing triangle, patients who reliably deteriorated; diamonds, patients who did not reliably change; dashed line remission threshold.

mindfulness skills and self-compassion (all $R^{2}>0.19$, all $F>99.1$ and $P<2 \times 10^{-16}$; Supplementary Fig. 3).

\section{Discussion}

This study was the first to examine the effectiveness of MBCT in patients with recurrent versus single episodes of MDD and current versus remitted MDD in a large, naturalistic uncontrolled cohort. Our main findings include high levels of treatment adherence in clinical practice and moderate-to-large effects of MBCT on depressive symptoms. Effectiveness did not significantly differ for MDD recurrence and remission status subgroups. Higher baseline levels of depressive symptoms and therapy adherence both predicted more depressive symptom reduction, whereas higher levels of psychiatric comorbidity and unemployment predicted less depressive symptom reduction.

Treatment adherence was found to be high, with $94 \%$ of patients following more than four sessions. When comparing MBCT adherence between the current sample and aforementioned RCTs, ${ }^{9,28,29}$ adherence ranged from $76 \%$ to $91 \%$ in aforementioned RCTs, compared with $94 \%$ in the current sample. We note that all patients received a clinical interview, during which motivation for MBCT

\begin{tabular}{|c|c|c|c|c|c|}
\hline Groups & Mean difference & $95 \% \mathrm{Cl}$ low & $95 \% \mathrm{Cl}$ high & Cohen's d & $n$ \\
\hline Recurrent episode, current & -8.28 & -9.71 & -6.84 & -0.76 & 179 \\
\hline Single episode, current & -8.21 & -11.11 & -5.31 & -0.83 & 48 \\
\hline Recurrent episode, remitted & -7.76 & -8.85 & -6.66 & -0.84 & 228 \\
\hline Single episode, remitted & -5.89 & -8.24 & -3.55 & -0.68 & 47 \\
\hline All & -7.81 & -8.60 & -7.02 & -0.75 & 502 \\
\hline
\end{tabular}


was discussed. It is a limitation of this study that we do not have solid quantitative data on how many patients who were referred did not proceed to MBCT following this interview. Based on our clinical experience with this population, we estimate this to be around 1 in 20 patients. For example, in 2017 around 300 patients were treated in MBCT groups at our centre, and only 11 patients did not start the training after clinical interview. In addition, lower education levels negatively affected treatment adherence. Those with lower education were underrepresented in the current sample. More efforts should be made to include these patients in MBCT and assess possible beneficial adaptations to benefit this group.

MBCT significantly decreased depressive symptoms with medium-to-large effect sizes. The current results are comparable with previous findings on the effectiveness of MBCT for depressive symptoms in real-world settings as conducted in the UK. ${ }^{30}$ Forty per cent of the patients that entered this study with Patient Health Questionnaire (PHQ-9) scores in the depressed range (total $n=828$; note that no formal psychiatric diagnoses was available in this study) showed reliable improvement, compared with $2 \%$ that showed reliable deterioration. These findings are remarkably similar to our findings in patients with an established diagnosis of MDD (40\% reliable change, $3 \%$ reliable deterioration). The fact that earlier efficacy results are maintained in clinical practice indicates that MBCT can be safely translated from highly specific and controlled research settings to the reality of clinical practice without compromising its effectiveness.

No significant differences in effectivity between MDD recurrence and remission subgroups could be established. To compare this finding in further detail to previous RCTs, we collected within-group effect sizes of the change in BDI-II scores in the MBCT arms of relevant previous RCTs. ${ }^{5,31}$ With regard to history of MDD, Tickell et $\mathrm{al}^{30}$ demonstrated a moderate within-group effect (Cohen's $d=0.31$ ) in patients with remitted, recurrent MDD with three or more episodes, whereas the effect size for remitted, recurrent MDD in our study was Cohen's $d=0.84$. With regard to remission status of MDD, a study of patients with chronic $\mathrm{MDD}^{28}$ found a moderate effect as well (Cohen's $d=0.48$; our sample: current/recurrent Cohen's $d=0.76$, current/single Cohen's $d=0.83$ ), Van Aalderen et $\mathrm{al}^{29}$ found moderate effects in both acute and remitted patients with MDD who suffered three or more previous episodes (Cohen's $d=0.71$ and 0.45 , respectively). In our sample, these effect sizes were similar and higher (current/ recurrent Cohen's $d=0.76$, recurrent/remitted Cohen's $d=0.84$ ).

That MBCT has comparable effects in patients with recurrent and single episodes of MDD in terms of depressive symptom reduction is in line with previous evidence suggesting that reduction of residual symptoms by MBCT is independent of number of previous episodes of MDD. ${ }^{13}$ In terms of remission status, our results are also in accordance with evidence from a recent meta-analysis ${ }^{5}$ indicating that MBCT was effective in the treatment of current depressive symptoms. The present results fill an important knowledge gap in terms of the effectiveness of MBCT in different clinical subpopulations of patients with depressive disorder as determined by psychiatric diagnosis. With regard to symptom reduction, our findings challenge the notion that MBCT should exclusively be offered to those with recurrent depression in remission.

Treatment success in terms of reduction of depressive symptoms was predicted by baseline symptom severity and, to a lesser extent, by employment status and psychiatric comorbidity. Unemployed patients demonstrated a markedly less favourable outcome than patients with planned daytime activities (i.e. paid work, students, homemakers) and patients who were on sick leave. This is in accordance with a previous meta-analysis indicating that socioeconomic deprivation is associated with poorer treatment outcomes in psychiatry in general. ${ }^{32}$ Qualitative research to better understand facilitators and barriers in the unemployed might lead to targeted interventions to improve outcome of MBCT in these populations. Moreover, higher levels of psychiatric (and in trend somatic) comorbidity were also associated with reduced beneficial effects of MBCT on depressive symptom reduction. Although this effect was small in terms of explained variance, we think it warrants more fine-grained investigation of specific comorbidities and their effect on treatment success of MBCT. Identifying specific MDD comorbidity profiles associated with less effect on depressive symptoms might readily lead to protocol changes.

\section{Strengths and limitations}

The current study is in line with the recommendation to engage more often in effectiveness research, focusing on external validity of interventions implemented by community providers under routine conditions in the real world'. ${ }^{7}$ Specific strengths of the current study are the large sample size, clinical representativeness, standardised psychiatric assessment and qualified MBCT teachers.

Important limitations to the study are inherent to routine outcome monitoring data in general, such as the lack of a control group. This implies that we cannot determine the specificity of the effects found. Moreover, the effect of self-selection by patients cannot be distilled from the current data-set, which might have resulted in selection bias. We also note that a third of patients did not fill out the post-treatment questionnaires. Patients with a comorbid personality disorder or developmental disorder were overrepresented in this group. Although the patients only slightly differed from the patients that did fill out the post-treatment questionnaires, we have to recognise this might reduce generalisability of our findings.

Other limitations are that a longer-term follow-up of the outcomes was not available. Lack of a follow-up for the semi-structured psychiatric interview rendered it impossible to determine clinical change in terms of recovery from depressive disorder. In addition, we did not systematically gather data on adverse events during MBCT. Although the meta-analysis by Kuyken et $\mathrm{al}^{4}$ indicates that adverse events related to the nature of the intervention are highly exceptional, we cannot substantiate this based on our data. In future studies, adverse events of mindfulness-based interventions should be monitored more carefully.

Overall, the current results illustrate that MBCT is an effective treatment in routine clinical practice for a heterogenic group of patients suffering from both recurrent and single MDD, and for those with either current or remitted depressive symptoms. MBCT can be translated from highly specific and controlled research settings to clinical practice without compromising its effectiveness. Notwithstanding the positive effects on depressive symptom reduction across all subgroups, we did observe lower adherence for those with low levels of education and less depressive symptom improvement for those without employment. Research to better understand facilitators and barriers in the unemployed and lower educated should lead to improved accessibility and outcome of MBCT, and should prevent MBCT from becoming an elitist treatment.

In summary, broad and inclusive implementation of this relatively short, group-based MBCT program might be an important contribution to relieving the burden of MDD for large populations in clinical practice.

Dirk E.M. Geurts (D), Department of Psychiatry, Radboud University; and Donders Institute for Brain, Cognition and Behavior, Radboud University, The Netherlands; Felix R. Compen, Department of Psychiatry, Radboud University; and Donders Institute for Brain, Cognition and Behavior, Radboud University, The Netherlands: Marleen H.C.T. Van Beek, Department of Psychiatry, Radboud University; and Donders 
Institute for Brain, Cognition and Behavior, Radboud University, The Netherlands; Anne E.M. Speckens, Department of Psychiatry, Radboud University; and Donders Institute for Brain, Cognition and Behavior, Radboud University, The Netherlands

Correspondence: Dr Dirk E.M. Geurts. Email: dirk.geurts@radboudumc.nl

First received 25 Aug 2020, final revision 23 Sep 2020, accepted 23 Sep 2020

\section{Supplementary material}

Supplementary material is available online at https://doi.org/10.1192/bjo.2020.118.

\section{Data availability}

The data that support the findings of this study are available from the corresponding author, D.E.M.G., upon reasonable request.

\section{Acknowledgements}

We thank our secretary Irma Veliscek-van Maren for organising the MBCT interventions and collecting routine outcome monitoring assessments; data manager Geert Schattenberg for importing the routine outcome monitoring data; and MBCT trainers Renée Metzemaekers, Martine Steen, Hetty Jansen, Rinie van den Bogaart, Hiske van Ravensteiin, Ellen Jansen and Ineke Hospers. We also thank Alexander van Daele, Isa van Dord, Lorraine Hagemeijer, Iris van Oostrom and Anna Kirschberger for helping with data import.

\section{Author contributions}

A.E.M.S. is the founder and clinical director of the centre where the diagnostic assessments and MBCT courses were provided. A.E.M.S. and D.E.M.G. supervised the clinical assessments, the MBCT courses and, together with M.H.C.T.V.B. and F.R.C., the collection of the routine outcome monitoring data. D.E.M.G. and F.R.C. were responsible for the analyses of the data. D.E.M.G. and F.R.C. drafted the paper, which has been edited by M.H.C.T.V.B. and A.E.M.S. All authors have read and approved the final version of the manuscript.

\section{Declaration of interest}

None.

ICMJE forms are in the supplementary material, available online at https://doi.org/10.1192/bjo. 2020.118 .

\section{References}

1 World Health Organization. Depression and Other Common Mental Disorders: Global Health Estimates. WHO, 2017 (https://apps.who.int/iris/bitstream/handle/10665/254610/WHO-MSD-MER-2017.2-eng.pdf)

2 Ferrari AJ, Charlson FJ, Norman RE, Patten SB, Freedman G, Murray CJ, et al. Burden of depressive disorders by country, sex, age, and year: findings from the global burden of disease study 2010. PLOS Med 2013; 10(11): e1001547.

3 Teasdale JD, Segal ZV, Williams JMG, Ridgeway VA, Soulsby JM, Lau MA. Prevention of relapse/recurrence in major depression by mindfulness-based cognitive therapy. J Consult Clin Psychol 2000; 68(4): 615-23.

4 Kuyken W, Warren FC, Taylor RS, Whalley B, Crane C, Bondolfi G, et al. Efficacy of mindfulness-based cognitive therapy in prevention of depressive relapse: an individual patient data meta-analysis from randomized trials. JAMA Psychiatry 2016; 73(6): 565-74.

5 Goldberg SB, Tucker RP, Greene PA, Davidson RJ, Kearney DJ, Simpson TL. Mindfulness-based cognitive therapy for the treatment of current depressive symptoms: a meta-analysis. Cogn Behav Ther 2019; 48(6): 445-62.

6 Rycroft-Malone J, Gradinger F, Owen Griffiths H, Anderson R, Crane RS, Gibson A, et al. 'Mind the gaps': the accessibility and implementation of an effective depression relapse prevention programme in UK NHS services: learning from mindfulness-based cognitive therapy through a mixed-methods study. BMJ Open 2019; 9(9): e026244.

7 Dimidjian S, Segal ZV. Prospects for a clinical science of mindfulness-based intervention. Am Psychol 2015; 70(7): 593-620.

8 Van Der Lem R, Van Der Wee NJ, Van Veen T, Zitman FG. Efficacy versus effectiveness: a direct comparison of the outcome of treatment for mild to moderate depression in randomized controlled trials and daily practice. Psychother Psychosom 2012; 81(4): 226-34.

9 Kuyken W, Hayes R, Barrett B, Byng R, Dalgleish T, Kessler D, et al. Effectiveness and cost-effectiveness of mindfulness-based cognitive therapy compared with maintenance antidepressant treatment in the prevention of depressive relapse or recurrence (PREVENT): a randomised controlled trial. Lancet 2015; 386 (9988): 63-73.
10 Crane RS, Kuyken W. The implementation of mindfulness-based cognitive therapy: learning from the UK health service experience. Mindfulness 2013; 4: 246-54.

11 Ma SH, Teasdale JD. Mindfulness-based cognitive therapy for depression: replication and exploration of differential relapse prevention effects. J Consult Clin Psychol 2004; 72(1): 31.

12 National Institute for Health and Care Excellence (NICE). Depression in Adults: Recognition and Management. Clinical Guideline [CG90]. NICE, 2009 (https:// www.nice.org.uk/guidance/cg90).

13 Geschwind N, Peeters F, Huibers M, van Os J, Wichers M. Efficacy of mindfulness-based cognitive therapy in relation to prior history of depression: randomised controlled trial. Br J Psychiatry 2012; 201(4): 320-5.

14 Strauss C, Cavanagh K, Oliver A, Pettman D. Mindfulness-based interventions for people diagnosed with a current episode of an anxiety or depressive disorder: a meta-analysis of randomised controlled trials. PLOS One 2014; 9(4): e96110.

15 Segal ZV, Williams JMG, Teasdale JD. Mindfulness-Based Cognitive Therapy for Depression: A New Approach to Relapse Prevention. Guilford Press, 2002.

16 UK Network of Mindfulness-Based Teacher Trainers. Good Practice Guidance for Teaching Mindfulness-Based Courses. Bangor University, 2010 (http:// www.bangor.ac.uk/mindfulness/documents/MBA\%20teacherGPG-Feb\%2010. pdf).

17 Comijs HC, van Marwijk HW, van der Mast RC, Naarding P, Voshaar RCO, Beekman AT, et al. The Netherlands study of depression in older persons (NESDO); a prospective cohort study. BMC Res Notes 2011; 4(1): 524.

18 Sheehan DV, Lecrubier $Y$, Sheehan $H$, Amorim $P$, Janavs J, Weiller E, et al. The Mini-International Neuropsychiatric Interview (MINI): the development and validation of a structured diagnostic psychiatric interview for DSM-IV and ICD-10. J Clin Psychiatry 1998; 59(suppl 20): 2233.

19 Beck AT, Steer RA, Brown GK. Beck Depression Inventory II (BDI-II). The Psychological Corporation, 1996.

20 Meyer TJ, Miller ML, Metzger RL, Borkovec TD. Development and validation of the Penn State Worry Questionnaire. Behav Res Ther 1990; 28(6): 487-95.

21 Van Rijsoort S, Emmelkamp P, Vervaeke G. The Penn State Worry Questionnaire and the worry domains questionnaire: structure, reliability and validity. Clin Psychol Psychother 1999; 6(4): 297-307.

22 Bohlmeijer E, ten Klooster PM, Fledderus M, Veehof M, Baer R. Psychometric properties of the Five Facet Mindfulness Questionnaire in depressed adults and development of a short form. Assessment 2011; 18(3): 308-20.

23 Raes F, Pommier E, Neff KD, Van Gucht D. Construction and factorial validation of a short form of the Self-Compassion Scale. Clin Psychol Psychother 2011; 18 (3): 250-5.

24 Kuznetsova A, Brockhoff PB, Christensen RHB. ImerTest package: tests in linear mixed effects models. J Stat Softw 2017; 82(13): 1-26.

25 Cohen J. A power primer. Psychol Bull 1992; 112(1): 155-9.

26 Jacobson NS, Truax P. Clinical significance: a statistical approach to defining meaningful change in psychotherapy research. J Consult Clin Psychol 1991; 59(1): 12-9.

27 Bland JM, Altman DG. Statistics notes: Cronbach's alpha. BMJ 1997; 314(7080): 572.

28 Cladder-Micus MB, Speckens AEM, Vrijsen JN, Donders ART, Becker ES, Spijker J. Mindfulness-based cognitive therapy for patients with chronic, treatmentresistant depression: a pragmatic randomized controlled trial. Depress Anxiety 2018; 35(10): 914-24.

29 Van Aalderen JR, Donders ART, Giommi F, Spinhoven P, Barendregt HP, Speckens AEM. The efficacy of mindfulness-based cognitive therapy in recurrent depressed patients with and without a current depressive episode: a randomized controlled trial. Psychol Med 2012; 42(5): 989-1001.

30 Tickell A, Ball S, Bernard P, Kuyken W, Marx R, Pack S, et al. The effectiveness of mindfulness-based cognitive therapy (MBCT) in real-world healthcare services. Mindfulness (N Y) 2020; 11(2): 279-90.

31 Goldberg SB, Tucker RP, Greene PA, Davidson RJ, Wampold BE, Kearney DJ, et al. Mindfulness-based interventions for psychiatric disorders: a systematic review and meta-analysis. Clin Psychol Rev 2018; 59: 52-60.

32 Finegan M, Firth N, Wojnarowski C, Delgadillo J. Associations between socioeconomic status and psychological therapy outcomes: a systematic review and meta-analysis. Depress Anxiety 2018; 35(6): 560-73. 